\title{
Gender Disparities of Heart Disease and the Association with Smoking and Alcohol Drinking Behavior Among the Middle-Aged and Older Population in the US and China, a Cross-Sectional Study of CHARLS and HRS Data
}

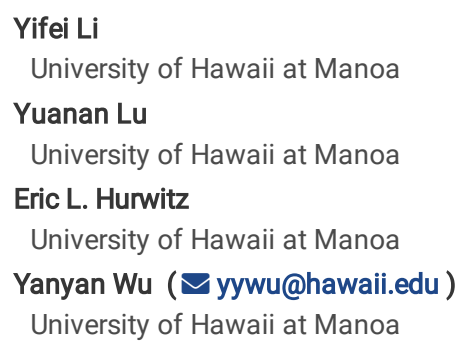




\section{Abstract \\ Background}

Heart disease remains the leading cause of death globally with substantial variabilities in mortalities by gender and region. Smoking and alcohol drinking are known modifiable health behaviors associated with heart disease. This study aims to estimate the prevalence of heart disease and to examine the association with smoking and drinking behavior for men and women in the United States (US) and China.

\section{Methods}

This study utilized the Harmonized data from the US Health and Retirement Study (HRS) and the China Health and Retirement Longitudinal Study (CHARLS), which are sister surveys as part of the Gateway to Global Aging Data (https://g2aging.org/). We performed cross-sectional comparisons using the 2016 wave HRS and 2015 wave CHARLS data. Age was categorized into four groups (50-59, 60-69, 70-79, and 80 years or older) and smoking and drinking behavior were combined to neither, smoking only, drinking only and both behaviors. Weighted analyses were conducted to estimate the prevalence and prevalence ratios (PRs) of heart disease accounting for complex survey design.

\section{Results}

The overall prevalence of heart disease was higher in men (24.5\%) than in women $(20.6 \%)$ in the US. In contrast, women had higher prevalence (22.9\%) than men (16.1\%) in China. The prevalence of heart disease increased by age with increasing gender gap in the US, while in China, the highest prevalence was observed in the 70-79 age group and gender difference were more apparent before 80 years of age. Adjusting for socio-demographic variables and health conditions, smoking only was associated with a higher prevalence of heart disease in both countries and the associations were stronger among women (US: $\mathrm{PR}=1.39,95 \% \mathrm{Cl}: 1.26$ to 1.54 ; China: $\mathrm{PR}=1.49,95 \% \mathrm{Cl}: 1.30$ to 1.72 ) than among men (US: $\mathrm{PR}=1.20,95 \% \mathrm{Cl}: 1.04$ to 1.38 ; China: $\mathrm{PR}=1.37,95 \% \mathrm{Cl}$ : 0.94 to 1.98).

\section{Conclusions}

Findings from this study will improve present understanding of heart disease etiology and provide essential insights for future prevention, treatment, and control. Better management of smoking behaviors by gender might be beneficial for reducing the burden of heart disease in both countries and worldwide.

\section{Introduction}

Heart disease remains the leading cause of death globally. The global evaluation of heart disease mortality showed that females had lower mortality rates of heart disease than males, and the differences declined with the increase of age [1]. The highest number of cardiovascular disease deaths occurred in China, followed by India, Russia, the U.S., and Indonesia [2]. It was estimated that the number of deaths due to cardiovascular disease was 17.9 million each year and anticipated to rise to 20 million by 2030 worldwide [3]. In the US, the age-adjusted mortality of heart disease in 2017 was 209.0 among men and 129.6 among women per 100,000 population [4]; in China, the rates reported in 2016 were 101.42 for men and 76.55 for women, respectively [5].

The burden of heart disease in both countries is closely related to the increased mortality, morbidity, and frailty in the affected individuals, which could also translate to significant overall healthcare costs [6]. Besides, population aging has brought similar public health challenges of heart disease in the US and China [7]. Older adults experienced higher rates of chronic diseases and were particularly vulnerable as heart disease-related morbidity and mortality both increased with age [8, 9]. According to the US National Center for Health Statistics, the prevalences of heart disease among men for different age groups were $9.5 \%$ (45-54 years), $16.7 \%$ (55-64 years), 29.8\% (65-74 years), $42.1 \%$ (75 years or older), while the prevalences among women were $9.4 \%$ ( $45-54$ years), $13 \%$ (55-64 years), 19.3\% (65-74 years) and 30.9\% (75 years or older) [4]. However, no specific statistics of heart disease prevalences by age groups or gender were found for China. A previous study showed that the mortality rate of heart disease among men for different age groups was 94.9 (45-64 years old) and 875.4 ( 65 + years old) per 100,000 population per year, while the mortality rate among women was 41.8 (45-64 years old) and 866.8 (65+years old) per 100,000 population per year in 2016 [5].

There are several risk factors for heart disease, which include socioeconomic status such as educational attainment; lifestyle behaviors such as alcohol use, cigarette smoking, unhealthy diet, restless sleep, and physical inactivity; other chronic diseases such as hypertension and diabetes; and body mass index (BMI) [10-12]. Several studies in multiple countries have shown that the mortality of coronary heart disease, the prevalences of cardiovascular major risk factors, as well as the health-related inequalities could be largely reduced by the change of unhealthy lifestyle behaviors [13-15]. Smoking and alcohol drinking are two commonly recognized unhealthy lifestyle behaviors in the US and China, and they are also among the top causes of preventable deaths [16]. Such behaviors not only pose health hazards to individuals but also increase the medical burdens to a greater extent. However, the management and control of these behaviors in the two countries are still below expectation. Therefore, the enhanced management of smoking and alcohol drinking behaviors could be an essential approach to reduce the unhealthy lifestyle-related chronic disease burden in our society [17]. Previous studies have shown that the associations between lifestyle behaviors and heart disease varied by age and gender. Coronary heart disease risk among current smokers was highest in the younger and lowest in the older participants in the US [18]. Gender differences existed in the effect-size measures of lifestyle-related factors, indicating that specific approaches were needed for men and women for enhanced prevention of primary and secondary cardiovascular disease management [19]. 
A cross-country study will improve the current understanding of the roles of age and gender in the relationship between smoking and alcohol drinking behaviors and heart disease. And new findings might provide essential baselines for the development of health promotion and health care strategies for the global population [1]. Therefore, this study aimed to estimate the prevalences of heart disease among middle-aged and older adults (aged 50-year-old and above) by gender, and analyze their associations with smoking/alcohol drinking behaviors in the US and China. We hypothesize that the associations of smoking and alcohol drinking behaviors and heart disease differ by age and gender groups in both countries.

\section{Methods \\ Data source}

This study used the Harmonized data from the US Health and Retirement Study (HRS) and the China Health and Retirement Longitudinal Study (CHARLS), which are sister surveys as part of the Gateway to Global Aging Data (https://g2aging.org/).

The HRS is a biennial longitudinal study that utilized a complex multi-stage area probability sampling design of the US populations aged 50 and over and their spouses [20, 21]. It has been conducted since 1992 and included a refresher cohort of 50-56 persons every 6 years. The HRS was sponsored by the National Institute of Aging (NIA U01AG009740) and is conducted by the University of Michigan. The data in HRS were collected by face-to-face interview or phone call, during which participants were asked questions about finances, health status and behaviors, marital/family status, and social support systems [21]. The CHARLS surveyed populations aged 45 years old and older and their spouses, including the assessments of social, economic, and health circumstances of community residents [22-24]. The baseline survey was conducted between June 2011 and March 2012, and participants were followed up every 2 years [25]. The study utilized the 2016 wave of HRS data from the RAND HRS 1992 to 2016 version 2 and the 2015 wave of Harmonized CHARLS version C and included those who were 50 years of age or older.

\section{Analytic sample}

The original sample sizes were 20,912 in the 2016 wave of HRS data and 20,281 in the 2015 wave of CHARLS data. The exclusion criteria of this study were: 1) participants aged less than 50 years old or missing age ( $n=764$ in HRS, $n=3,860$ in CHARLS); 2$)$ missing heart disease status ( $n=40$ in HRS, $n=1,560$ in CHARLS); 3) missing of both smoking and alcohol drinking ( $n=100$ in HRS, $n=37$ in CHARLS); and 4) observations with missing data on diabetes or high blood pressure ( $n=76$ in HRS, $n=495$ in CHARLS). The sample sizes employed in this study were 19,932 in the HRS and 14,329 in CHARLS. The detailed sampling flow charts are shown in Fig. 1.

\section{Variables}

The response variable heart disease was defined by the respondents' self-reported answers to the question: "Has a doctor ever told you that you have had a heart attack, coronary heart disease, angina, congestive heart failure, or other heart problems?" The answer was coded as "Yes" or "No".

The exposure variable was smoking and alcohol drinking status, which was categorized as "Smoking and drinking”, "Only smoking", "Only drinking”, and "Neither". Smoking and alcohol drinking behaviors were combined measure from the questions that whether the respondent reported ever smoking, and ever drinking. The definition of smoking included more than 100 cigarettes in the lifetime in the US, and chewing tobacco, smoking a pipe, self-rolled cigarettes, cigarettes, or cigars in China. The drinking types referred to any kind of alcoholic beverages in the US and various alcoholic beverages, including white liquor and liang of liquor in China.

The gender differences in prevalences of heart disease and the gender differences in smoking and alcohol drinking behaviors were previously found according to the literature reviews. For our study, gender was regarded as a potential effect modifier and was coded as "Men" and "Women". Further analyses of the effect estimates were compared based on the gender stratification in both countries.

Confounding variables included in the study were: age group (50-59, 60-69, 70-70, and $\geq 80$ years old), BMI (underweight/normal: $<25 \mathrm{~kg} / \mathrm{m}^{2}$, overweight: $25 \leq \mathrm{BMl}<30 \mathrm{~kg} / \mathrm{m}^{2}$, and obese: $\geq 30 \mathrm{~kg} / \mathrm{m}^{2}$ ), education (less than high school, high school or equivalent (high school or vocational school), and the associate degree or higher (some college, associate degree, bachelor's degree and above)), sleep quality (restless or good), high blood pressure, and diabetes. Restless sleep was defined as how often had trouble to fall asleep most of the time in the HRS and $\geq 3$ days a week in CHARLS. Similar to heart disease, high blood pressure and diabetes were self-reported doctor-diagnosed conditions. There was substantial missingness in CHARLS sleep quality and BMI data, therefore we coded the missingness as a category "did not report" for both surveys to ensure the population representativeness of the data.

\section{Statistical analysis}

Statistical software R version 3.5.2 was used for the analyses and all analyses account for complex survey weights. Separate analyses were performed for men and women in the US and China, respectively. We first computed descriptive statistics (frequency and weighted percentage) to summarize the sample characteristics. Next, we computed the weighted prevalences of heart disease by age group and smoking/drinking behavior, and the corresponding $95 \%$ confidence intervals (Cls) by country. Lastly, modified Poisson regression analyses were conducted to estimate the weighted crude prevalence ratio (cPR) and adjusted prevalence ratio (adjPR) of heart disease by age group (reference: 50-59 years) and drinking behavior (reference: neither smoking nor drinking), with the corresponding $95 \% \mathrm{Cls}$. The adjPRs were estimated by controlling for BMI, educational level, sleep quality, high blood pressure, and diabetes.

\section{Results}

\section{Sample characteristics}


As shown in Table 1, there were $46.6 \%$ men in the US HRS sample and $48.7 \%$ men in the CHARLS sample. In the US, $40.7 \%$ of men and $31.3 \%$ of women reported yes to both smoking and drinking behaviors, and $12.8 \%$ of men and $24.3 \%$ of women reported no to neither behavior. As for China, $61.1 \%$ of men and only $2.9 \%$ of women reported both smoking and drinking, while $6.9 \%$ of men and $71.1 \%$ of women reported neither behavior. The overall prevalences of heart disease were $24.5 \%$ among men and $20.6 \%$ among women in the US, and $16.1 \%$ among men, and $22.9 \%$ among women in China.

Table 1

Sample size (N) and weighted percentages (wt\%) by gender and country.

\begin{tabular}{|c|c|c|c|c|}
\hline \multirow[t]{3}{*}{ Variable } & \multicolumn{2}{|c|}{ US $(N=19932)$} & \multicolumn{2}{|c|}{ China $(N=14329)$} \\
\hline & Men $(46.6 \%)$ & Women $(53.4 \%)$ & Men (48.7\%) & Women (51.3\%) \\
\hline & $N(w t \%)$ & $N(w t \%)$ & $N(w t \%)$ & $N(w t \%)$ \\
\hline \multicolumn{5}{|l|}{ Age group (years) } \\
\hline $50-59$ & $2980(38.6)$ & $3868(34.8)$ & $2695(38.7)$ & $2946(40.2)$ \\
\hline $60-69$ & $2492(33.7)$ & $3363(33.5)$ & $2671(37.2)$ & $2747(35.7)$ \\
\hline $70-79$ & $1720(18.4)$ & $2432(18.8)$ & $1293(18.3)$ & $1219(17.0)$ \\
\hline $80+$ & 1203 ( 9.3$)$ & $1874(12.9)$ & $340(5.8)$ & $418(7.1)$ \\
\hline Median and maximum & 64 and 103 & 65 and 107 & 62 and 101 & 62 and 105 \\
\hline \multicolumn{5}{|l|}{ Smoking and drinking behavior } \\
\hline Neither & $1151(12.8)$ & $3204(24.3)$ & 449 ( 6.9) & $5224(71.1)$ \\
\hline Only drinking & $2019(28.1)$ & $2703(26.5)$ & $732(10.2)$ & $1380(19.3)$ \\
\hline Only smoking & $1750(18.4)$ & $2283(17.9)$ & $1538(21.8)$ & $505(6.7)$ \\
\hline Both & $3475(40.7)$ & $3347(31.3)$ & $4280(61.1)$ & $221(2.9)$ \\
\hline \multicolumn{5}{|l|}{ Education } \\
\hline Less than high school & $1460(11.9)$ & $1998(12.5)$ & $5836(82.3)$ & $6743(91.7)$ \\
\hline High school or equivalent & $4207(48.4)$ & $6141(51.6)$ & $946(14.5)$ & $514(7.0)$ \\
\hline Associate degree or higher & $2728(39.7)$ & $3398(35.9)$ & $217(3.1)$ & $73(1.3)$ \\
\hline \multicolumn{5}{|l|}{ High blood pressure } \\
\hline No & $3236(42.7)$ & $4475(45.3)$ & $4551(64.4)$ & $4533(61.9)$ \\
\hline Yes & $5159(57.3)$ & $7062(54.7)$ & $2448(35.6)$ & $2797(38.1)$ \\
\hline \multicolumn{5}{|l|}{ Diabetes } \\
\hline No & $6013(75.2)$ & 8479 (77.5) & $6317(90.2)$ & $6430(87.8)$ \\
\hline Yes & $2382(24.8)$ & $3058(22.5)$ & $682(9.8)$ & $900(12.2)$ \\
\hline \multicolumn{5}{|l|}{ Sleep quality } \\
\hline Restless & $1729(21.1)$ & $2901(25.0)$ & $1755(24.4)$ & $2998(39.4)$ \\
\hline Good & $6614(78.4)$ & $8572(74.6)$ & 4839 (69.3) & 3851 (52.7) \\
\hline Did not report & $52(0.5)$ & $64(0.5)$ & 405 ( 6.3$)$ & $481(7.9)$ \\
\hline \multicolumn{5}{|l|}{$B M I$} \\
\hline Underweight or normal & $1862(20.8)$ & $3364(31.4)$ & $3898(54.1)$ & 3707 (50.6) \\
\hline Overweight & 3571 (43.6) & $3563(30.7)$ & $1423(20.8)$ & $1856(24.2)$ \\
\hline Obese & $2907(35.1)$ & $4368(36.0)$ & $191(2.7)$ & $397(4.9)$ \\
\hline Did not report & $55(0.5)$ & $242(1.9)$ & $1487(22.5)$ & $1370(20.3)$ \\
\hline \multicolumn{5}{|l|}{ Heart disease } \\
\hline No & $6190(75.5)$ & 8969 (79.4) & $5833(83.9)$ & $5640(77.1)$ \\
\hline Yes & $2205(24.5)$ & 2568 (20.6) & $1166(16.1)$ & $1690(22.9)$ \\
\hline
\end{tabular}

\section{Prevalences of heart disease by age group and smoking/drinking behavior}

Figure 2 shows the prevalences of heart disease with $95 \%$ Cls by age group and smoking and drinking behaviors by gender for both countries. Nonoverlapping 95\% Cls between groups implies statistically significant differences (the prevalences and 95\% Cls can be found in Supplementary Table S1). The 
patterns differed by country. In the US, the prevalences of heart disease increased linearly with age, and men had higher prevalences (men: $12.9-53 \%$ and women: $11.3-39.7 \%$ from 50 to $80+$ ). The gender differences were more apparent in older age groups. While in China, the prevalences of heart disease were higher in women and had a nonlinear pattern with age with the highest prevalence in the $70-79$ age group (men: $10.1-24.5 \%$ and women: $17.1-30.6 \%$ from 50 to $80+$ ). The gender differences in China were more apparent in younger age groups.

Smoking had the strongest impact on both men and women in the US but only on women in China. For those who only smoked in the US, the heart disease prevalences were the highest (35.5\% for men and 31.5\% among women) as compared to other behavior groups. And drinking behavior was associated with a lower prevalence of heart disease for both men and women in the US. In China, no association was found between prevalence of heart diseases and smoking and drinking behaviors in men, however, smoking was associated with 15\% higher prevalence of heart disease among women (smoking and drinking: $34.2 \%$ and only smoking: $34.4 \%$ versus neither smoking nor drinking: $21.7 \%$ and only drinking: $21.8 \%$ ).

\section{Prevalence ratios of heart disease by age group and smoking/drinking behavior}

Table 2 shows the weighted prevalence ratio (adjPR) of heart disease, 95\% Cls and $p$-values by age and smoking/drinking behavior adjusting for education, diabetes, high blood pressure, sleep quality, and BMI categories. Crude PRs and adjusted PRs for all variables are presented in Supplementary Tables S2 and S3.

In the US, only smoking was associated with a higher prevalence of heart disease, the adjPR was 1.20 in men $(95 \% \mathrm{Cl}: 1.04,1.38, p=0.015)$ and 1.39 in women (95\% Cl: $1.26,1.54, p \leq 0.001)$ compared with those who did not smoke or drink before. Only drinking was associated with lower prevalence of heart disease, the adjPR was 0.74 in men ( $95 \% \mathrm{Cl}: 0.62,0.87, p<0.001)$ and 0.91 in women $(95 \% \mathrm{Cl}: 0.77,1.07, p=0.248)$.

After adjusting for all confounders, only smoking was found to have a marginal association with heart disease for men in China (adjPR: $1.37,95 \%$ Cl: 0.94 , $1.98, p=0.098)$ as opposed to the crude analysis of prevalences. The adjPR in women who only smoked was $1.49(95 \% \mathrm{Cl}: 1.30,1.72, p \leq 0.001)$. Among those who reported both smoking and drinking, the adjPRs were $1.28(95 \% \mathrm{Cl}: 0.91,1.78, p=0.149)$ in men and $1.54(95 \% \mathrm{Cl}$ : $0.26,1.89, p \leq 0.001)$ in women.

Table 2

Weighted prevalence ratio (adjPR) of heart disease, $95 \%$ Cls and $p$-values by age and smoking/drinking behavior adjusting for education, diabetes, high blood pressure, sleep

\begin{tabular}{|c|c|c|c|c|c|}
\hline \multirow[t]{2}{*}{ Country } & \multirow[t]{2}{*}{ Variables } & \multicolumn{2}{|l|}{ Men } & \multicolumn{2}{|l|}{ Women } \\
\hline & & $\operatorname{adjPR}(95 \% \mathrm{Cl})$ & $\mathbf{p}$ & $\operatorname{adjPR}(95 \% \mathrm{Cl})$ & $\mathbf{p}$ \\
\hline \multirow[t]{10}{*}{ US } & \multicolumn{5}{|l|}{ Age (years) } \\
\hline & $50-59$ & Ref & & Ref & \\
\hline & $60-69$ & $1.59(1.37,1.84)$ & $<0.001$ & $1.55(1.31,1.83)$ & $<0.001$ \\
\hline & $70-79$ & $2.23(1.95,2.56)$ & $<0.001$ & $2.00(1.73,2.31)$ & $<0.001$ \\
\hline & $80+$ & $3.34(2.91,3.84)$ & $<0.001$ & $2.78(2.39,3.24)$ & $<0.001$ \\
\hline & \multicolumn{5}{|c|}{ Smoking/drinking behavior } \\
\hline & Neither & Ref & & Ref & \\
\hline & Only drinking & $0.74(0.62,0.87)$ & $<0.001$ & $0.91(0.77,1.07)$ & 0.248 \\
\hline & Only smoking & $1.20(1.04,1.38)$ & 0.015 & $1.39(1.26,1.54)$ & $<0.001$ \\
\hline & Both & $1.01(0.89,1.15)$ & 0.877 & $0.98(0.86,1.11)$ & 0.725 \\
\hline \multirow[t]{10}{*}{ China } & \multicolumn{5}{|l|}{ Age (years) } \\
\hline & $50-59$ & Ref & & Ref & \\
\hline & $60-69$ & $1.67(1.39,1.99)$ & $<0.001$ & $1.36(1.19,1.55)$ & $<0.001$ \\
\hline & $70-79$ & $2.28(1.92,2.70)$ & $<0.001$ & $1.49(1.28,1.73)$ & $<0.001$ \\
\hline & $80+$ & $1.91(1.36,2.69)$ & $<0.001$ & $1.30(1.03,1.65)$ & 0.025 \\
\hline & \multicolumn{5}{|c|}{ Smoking/drinking behavior } \\
\hline & Neither & Ref & & Ref & \\
\hline & Only drinking & $1.26(0.88,1.81)$ & 0.212 & $1.00(0.85,1.18)$ & 0.953 \\
\hline & Only smoking & $1.37(0.94,1.98)$ & 0.098 & $1.49(1.30,1.72)$ & $<0.001$ \\
\hline & Both & $1.28(0.92,1.78)$ & 0.149 & $1.54(1.26,1.89)$ & $<0.001$ \\
\hline
\end{tabular}

\section{Discussion}


This cross-sectional study estimated the prevalence disparities of heart disease among older men and women with smoking/alcohol drinking behaviors in the US and China. Different patterns of association were found with age (linear in the US and nonlinear in China). A higher prevalence of heart disease was observed among men in the US but among women in China. Smoking only was found to be associated with a higher prevalence of heart disease for both men and women in both countries. Our results underline that better management of smoking would be crucial for health promotion and heart disease prevention.

The pattern of the increasing prevalence of heart disease by age and gender differences found in the HRS sample was similar to the US National Center for Health Statistics [4]. The decreased prevalence of heart disease among the $80+$ age group in CHARLS might partially be due to the lower life expectancy in China and the historical background for this group. Based on the birth cohort, the CHARLS participants who were aged no less than 80 in 2016 were born in 1936 and before, this age group might have lower health status since they had experienced and been affected by the unstable society and survived from the background of wars, social chaos and famine [26]. Additionally, the sample sizes of age $80+$ in the CHARLS were relatively small and only accounted for $5.8 \%$ of older men $(\mathrm{N}=340)$ and $7.1 \%$ of women $(\mathrm{N}=418)$, in comparison to $9.3 \%(\mathrm{~N}=1203)$ US older women and $12.9 \%(\mathrm{~N}=1874)$ older men.

The opposite gender differences in CHARLS may be explained by historical background and the gender inequality of China. The previous literature showed that older women in China always had lower social status and were undereducated with lower income levels [7]. Similar statistics were also observed from our study that more than $90 \%$ of women in China had lower than high school educational level, and the higher percentage of high blood pressure, as well as diabetes, were also observed among women when compared with men (Table 1). We found far fewer women than men reported smoking, which might be related to the health inequities and social backgrounds. In the traditional culture, smoking and drinking were discouraged for women, most women in the older generations needed to comply with the traditional gender role [27]. Although with the rapid socio-economic changes in the last few decades, women's social status has improved, such changes might also bring great pressure and poor mental health conditions for older women [28]. We also found the prevalence of heart diseases was higher among women who reported smoking compared with men who reported smoking (Fig. 2), which might be related to the reality that older women were more likely to be underinsured and lack the ability to access the social health insurance for self-health care [29]. The heavy impacts of smoking on heart disease among women in China found in our study indicate that to better control heart disease, future health care strategies should address such unhealthy behavior among women.

These findings support our hypothesis that smoking behavior is associated with a higher prevalence of heart disease and the association differs by gender and country. Smoking was associated with heart disease prevalence with a stronger effect on women, especially for the women in China, which was consistent with a previous literature that women who smoked in China had higher mortality and worse prognosis after acute cardiovascular events [30]. The impact of smoking on heart disease was also reported by Tolstrup et al. [18]. They utilized pooled datasets and demonstrated that smoking control was essential for heart disease prevention, and the majority of heart disease was attributable to smoking for all age groups [18]. Therefore, the important impacts of smoking behavior on heart disease among older populations cannot be ignored according to our findings, which elicits the recommendation that older populations in the US and China should be encouraged to reduce cigarette consumption to prevent and control heart disease and also improve their quality of life.

In addition, this study found a significantly lower adjPR of heart disease for those with only drinking behavior among men in the US. Abat et al. have recently demonstrated that alcohol consumption levels were associated with aging [31]. The relationships between alcohol drinking and heart disease was dependent on the amount to be consumed in daily life, and the light to moderate alcohol consumption was safe and would be beneficial to the cardiovascular system [32, 33]. However, several other researchers defended the harmful effects of alcohol intake, even at a low consumption level, stating that it outweighed their benefits $[34,35]$. Thus, the estimated impacts of drinking might be misleading due to the unaccounted consumption amounts and frequency of drinking in this study. Therefore, it would be safe to recommend that aligns with previous studies to keep a light to moderate alcohol intake among the current drinkers and to continue no drinking for non-drinkers for the health improvement and heart disease prevention $[31,33,36]$.

This study was conducted based on the relatively large sample size from two national-level surveys in the US and China. The results would be comparable since this study utilized harmonized datasets with similar years in both countries, and the findings might be generalizable to other older populations. However, this study has several limitations. First, both surveys in the US and China were based on self-reported data, it might have information biases such as misclassification due to recall biases of specific chronic disease conditions. The prevalences of heart disease might be underestimated, and the association disparities by countries might have been attenuated if the recall bias not occurred. Second, there are several differences between the countries that may have affected our estimates. According to the data manipulation, we used the same cut-off of BMI categories in both countries ignoring the fact that people in China usually have lower BMI than those in the US. Although our regression analysis results found no differences with/without the adjustment of BMI (see supplementary table S3 and S4), sensitivity analysis of different BMI cut-off for China by gender should be conducted in the future. Nonetheless, although harmonized datasets were used, the specific drinking types and the educational level categories were not the same in both countries. For instance, there were specific types of alcohol drinking such as liang of liquor in China, which was not included in the survey of the US; and an essential proportion of participants in China was illiterate and had no educational experiences, but no comparable groups could be chosen in the US for the estimates of prevalence disparities among illiterate populations in both countries. Therefore, more studies are needed to specify the drinking and education impacts on the prevalence disparities of heart disease in both countries. Third, there were some potential but unmeasured confounding variables in this study, such as race, physical activity, mental health status, types and dosage of smoking and alcohol consumption, income level, and nutritional status. There were no ethnicity or race categories in the CHARLS dataset, and the income level, nutrition status as well as mental health status may not be comparable between the two countries. Physical activity was an important predictor of heart disease but was not included in this study. It was because only a randomized half of participants were selected to measure physical activity status in China, the sample size would be extremely small if the physical activity variable were included as a confounder in the statistical analyses. Future studies should consider these potential confounders to estimate the association disparities of heart disease among the old populations by country. Fourth, since the study design was cross-sectional, causal inferences are limited. Only prevalences of heart disease could be observed in the cross-sectional analyses while the incidence of heart disease and its association with smoking and alcohol drinking behaviors were unable to be 
determined in the current study. Cohort studies are needed to identify the impacts of smoking and alcohol drinking behaviors on heart disease incidence and mortality, especially for the comparisons between the older populations in the US and China.

\section{Conclusion}

This study was conducted by utilizing datasets from two national surveys in the US and China, and the results show that the prevalence disparities of heart disease exist between smoking only and various age groups by gender in both countries. The findings might form the baseline information for the development of earlier control and management of smoking consumption, which would be an important strategy for health promotion and reducing the burden of heart disease in both the US and China.

\section{Abbreviations}

US: United States

HRS: Health and Retirement Study

CHARLS: China Health and Retirement Longitudinal Study

CPR: crude prevalence ratio

adjPR: adjusted prevalence ratio

BMl: body mass index

Cl: confidence interval

\section{Declarations}

\section{Ethics approval and consent to participate}

This study utilized publicly available secondary data. According to the US National Institute of Health, institutional approval is not required. All procedures performed in studies involving human participants were in accordance with the ethical standards of the institutional and/or national research committee and with the 1964 Helsinki declaration and its later amendments or comparable ethical standards. The original CHARLS was approved by the Ethical Review Committee of Peking University, and all participants signed informed consent at the time of participation.

\section{Consent for publication}

Not applicable.

\section{Availability of data and materials}

The datasets generated and analyzed during the current study are available in the GATEWAY to GLOBAL AGING DATA website, available in https://g2aging.org/.

\section{Competing interests}

The authors declare that there are no conflicts of interest.

\section{Funding}

This study did not receive any specific grant from funding agencies in the public, commercial, or not-for-profit sectors. The Harmonized datasets were developed by the Gateway to Global Aging Data and were funded by the National Institute on Aging (R01 AG030153, RC2 AG03669, 1R03AG043052).

\section{Author information}

\section{Affiliation}

Office of Public Health Studies, University of Hawai i at Mānoa, Hawaii, US

Yifei Li

Office of Public Health Studies, University of Hawai i at Mānoa, Hawaii, US

Yanyan Wu

Office of Public Health Studies, University of Hawai i at Mānoa, Hawaii, US

Yuanan Lu 
Office of Public Health Studies, University of Hawai i at Mānoa, Hawaii, US

Eric L. Hurwitz

\section{Contributions}

Y.F.L., Y.Y.W, and E.L.H. conceptualized and designed the study. Y.F.L. and Y.Y.W. performed the data analysis, and have full access to all the data in this study, and takes responsibility for the integrity of the data and the accuracy of the data analysis. Y.F.L. and Y.Y.W. drafted the manuscript, and were the major contributors in writing the manuscript. Y.A.L. and E.L.H. provided supervision of the project. Y.F.L., Y.Y.W., Y.A.L., and E.L.H. contributed to the data interpretation and revision of the manuscript. All the authors read and approved the final manuscript.

\section{Corresponding author}

Correspondence to Yanyan Wu

\section{Acknowledgements}

For CHARLS, we thank the China Center for Economic Research, the National School of Development of Peking University for providing the data [24]. We thank the HRS and CHARLS research and field teams and every respondent in the study for their contributions.

\section{References}

1. Bots SH, Peters SAE, Woodward M. Sex differences in coronary heart disease and stroke mortality: a global assessment of the effect of ageing between 1980 and 2010. BMJ Glob Health [Internet]. 2017 Mar 27 [cited 2021 May 10];2(2). Available from:

https://www.ncbi.nlm.nih.gov/pmc/articles/PMC5435266/

2. Roth GA, Mensah GA, Johnson CO, Addolorato G, Ammirati E, Baddour LM, et al. Global Burden of Cardiovascular Diseases and Risk Factors, 1990-2019: Update From the GBD 2019 Study. Journal of the American College of Cardiology. 2020 Dec 22;76(25):2982-3021.

3. Mathers CD, Loncar D. Projections of global mortality and burden of disease from 2002 to 2030. PLoS Med. 2006 Nov;3(11):e442.

4. Data Finder - Health, United States - Products [Internet]. National Center for Health Statistics. 2021 [cited 2021 Jun 1]. Available from: https://www.cdc.gov/nchs/data/hus/2019/005-508.pdf

5. Zhu J, Cui L, Wang K, Xie C, Sun N, Xu F, et al. Mortality pattern trends and disparities among Chinese from 2004 to 2016. BMC Public Health [Internet]. 2019 Sep 2 [cited 2021 Jun 2];19. Available from: https://www.ncbi.nlm.nih.gov/pmc/articles/PMC6717976/

6. Yoon SSS, Dillon CF, Illoh K, Carroll M. Trends in the Prevalence of Coronary Heart Disease in the U.S.: National Health and Nutrition Examination Survey, 2001-2012. Am J Prev Med. 2016 Oct;51(4):437-45.

7. Li H, Ge S, Greene B, Dunbar-Jacob J. Depression in the context of chronic diseases in the United States and China. Int J Nurs Sci. 2018 Nov 29;6(1):11722.

8. Rosenwohl-Mack A, Schumacher K, Fang M-L, Fukuoka Y. Experiences of aging in place in the United States: protocol for a systematic review and metaethnography of qualitative studies. Syst Rev. 2018 Oct 6;7(1):155.

9. Stone CL. A population-based measure of chronic disease severity for health planning and evaluation in the United States. AIMS Public Health. 2020 Feb 4;7(1):44-65.

10. Report on Cardiovascular Diseases in China 2018.pdf [Internet]. [cited 2021 May 31]. Available from:

https://www.nccd.org.cn/Sites/Uploaded/File/2021/3/\%E4\%B8\%AD\%E5\%9B\%BD\%E5\%BF\%83\%E8\%A1\%80\%E7\%AE\%A1\%E7\%97\%85\%E6\%8A\%A5\%E5\%9

11. CDC. Heart Disease | cdc.gov [Internet]. Centers for Disease Control and Prevention. 2021 [cited 2021 May 10]. Available from: https://www.cdc.gov/heartdisease/index.htm

12. Diabetes, Heart Disease, and Stroke | NIDDK [Internet]. [cited 2021 May 10]. Available from: https://www.niddk.nih.gov/healthinformation/diabetes/overview/preventing-problems/heart-disease-stroke

13. Han C, Liu F, Yang X, Chen J, Li J, Cao J, et al. Ideal cardiovascular health and incidence of atherosclerotic cardiovascular disease among Chinese adults: the China-PAR project. Sci China Life Sci. 2018 May;61(5):504-14.

14. Li Y, Wang DD, Ley SH, Howard AG, He Y, Lu Y, et al. Potential Impact of Time Trend of Life-Style Factors on Cardiovascular Disease Burden in China. J Am Coll Cardiol. 2016 Aug 23;68(8):818-33.

15. Lee I, Kim S, Kang H. Lifestyle Risk Factors and All-Cause and Cardiovascular Disease Mortality: Data from the Korean Longitudinal Study of Aging. Int J Environ Res Public Health [Internet]. 2019 Sep [cited 2021 May 10];16(17). Available from: https://www.ncbi.nlm.nih.gov/pmc/articles/PMC6747152/

16. Mokdad AH, Marks JS, Stroup DF, Gerberding JL. Actual causes of death in the United States, 2000. JAMA. 2004 Mar 10;291(10):1238-45.

17. Shah A, Shah J, Shah N, Shah R. Smoking prevalence and chronic diseases in China. The Lancet Respiratory Medicine. 2019 Mar 1;7(3):e10.

18. Tolstrup JS, Hvidtfeldt UA, Flachs EM, Spiegelman D, Heitmann BL, Bälter K, et al. Smoking and Risk of Coronary Heart Disease in Younger, Middle-Aged, and Older Adults. Am J Public Health. 2014 Jan;104(1):96-102.

19. Kouvari M, Panagiotakos DB, Chrysohoou C, Georgousopoulou E, Notara V, Tousoulis D, et al. Gender-specific, Lifestyle-related Factors and 10-year Cardiovascular Disease Risk; the ATTICA and GREECS Cohort Studies. Curr Vasc Pharmacol. 2019;17(4):401-10. 
20. Health and Retirement Study Data File [Internet]. [cited 2021 May 10]. Available from: https://www.rand.org/well-being/social-and-behavioralpolicy/centers/aging/dataprod/hrs-data.html

21. Thompson MD, Pirkle CM, Youkhana F, Wu YY. Gene-Obesogenic Environment Interactions on Body Mass Indices for Older Black and White Men and Women from the Health and Retirement Study. Int J Obes (Lond). 2020 Sep;44(9):1893-905.

22. Hua J. Stratum Differences in Health Behaviour of Elderly Group-An empirical research based on harmonized CHARLS. Journal of Physics: Conference Series. 2020 Jan 1;1437:012114.

23. Zhao Y, Hu Y, Smith JP, Strauss J, Yang G. Cohort Profile: The China Health and Retirement Longitudinal Study (CHARLS). Int J Epidemiol. 2014 Feb;43(1):61-8.

24. Zhao Y, Strauss J, Yang G, Giles J, Hu P; Hu Y, et al. China Health and Retirement Longitudinal Study, 2011-2012 National Baseline Users' Guide, National School of Development, Peking University. 2013

25. Ding L, Liang Y, Tan ECK, Hu Y, Zhang C, Liu Y, et al. Smoking, heavy drinking, physical inactivity, and obesity among middle-aged and older adults in China: cross-sectional findings from the baseline survey of CHARLS 2011-2012. BMC Public Health. 2020 Jul 6;20(1):1062.

26. Timeline of Events in China | Facing History and Ourselves [Internet]. [cited 2021 Jun 4]. Available from: https://www.facinghistory.org/nanjing-atrocitiescrimes-war/timeline-events-china

27. Cui Y, Zhu Q, Lou C, Gao E, Cheng Y, Zabin LS, et al. Gender differences in cigarette smoking and alcohol drinking among adolescents and young adults in Hanoi, Shanghai, and Taipei. J Int Med Res. 2018 Dec 1;46(12):5257-68.

28. Chuang Y-C, Chuang K-Y. Gender differences in relationships between social capital and individual smoking and drinking behavior in Taiwan. Soc Sci Med. 2008 Oct;67(8):1321-30.

29. Zhou M, Zhao S, Zhao Z. Gender differences in health insurance coverage in China. International Journal for Equity in Health. 2021 Feb 1;20(1):52.

30. Gao Z, Chen Z, Sun A, Deng X. Gender differences in cardiovascular disease. Medicine in Novel Technology and Devices. 2019 Dec 1;4:100025.

31. Abat C, Roussel Y, Chaudet H, Raoult D. Alcohol and the global burden of disease. The Lancet. 2019 Jun;393(10189):2390-1.

32. Rehm J, Roerecke M. Cardiovascular effects of alcohol consumption. Trends in Cardiovascular Medicine. 2017 Nov 1;27(8):534-8.

33. Chiva-Blanch G, Badimon L. Benefits and Risks of Moderate Alcohol Consumption on Cardiovascular Disease: Current Findings and Controversies. Nutrients. 2020 Jan;12(1):108.

34. Piepoli MF, Abreu A, Albus C, Ambrosetti M, Brotons C, Catapano AL, et al. Update on cardiovascular prevention in clinical practice: A position paper of the European Association of Preventive Cardiology of the European Society of Cardiology. Eur J Prev Cardiol. 2020 Jan;27(2):181-205.

35. Arnett DK, Blumenthal RS, Albert MA, Buroker AB, Goldberger ZD, Hahn EJ, et al. 2019 ACC/AHA Guideline on the Primary Prevention of Cardiovascular Disease: Executive Summary: A Report of the American College of Cardiology/American Heart Association Task Force on Clinical Practice Guidelines. J Am Coll Cardiol. 2019 Sep 10;74(10):1376-414.

36. Kloner Robert A., Rezkalla Shereif H. To Drink or Not to Drink? That Is the Question. Circulation. 2007 Sep 11;116(11):1306-17.

\section{Figures}




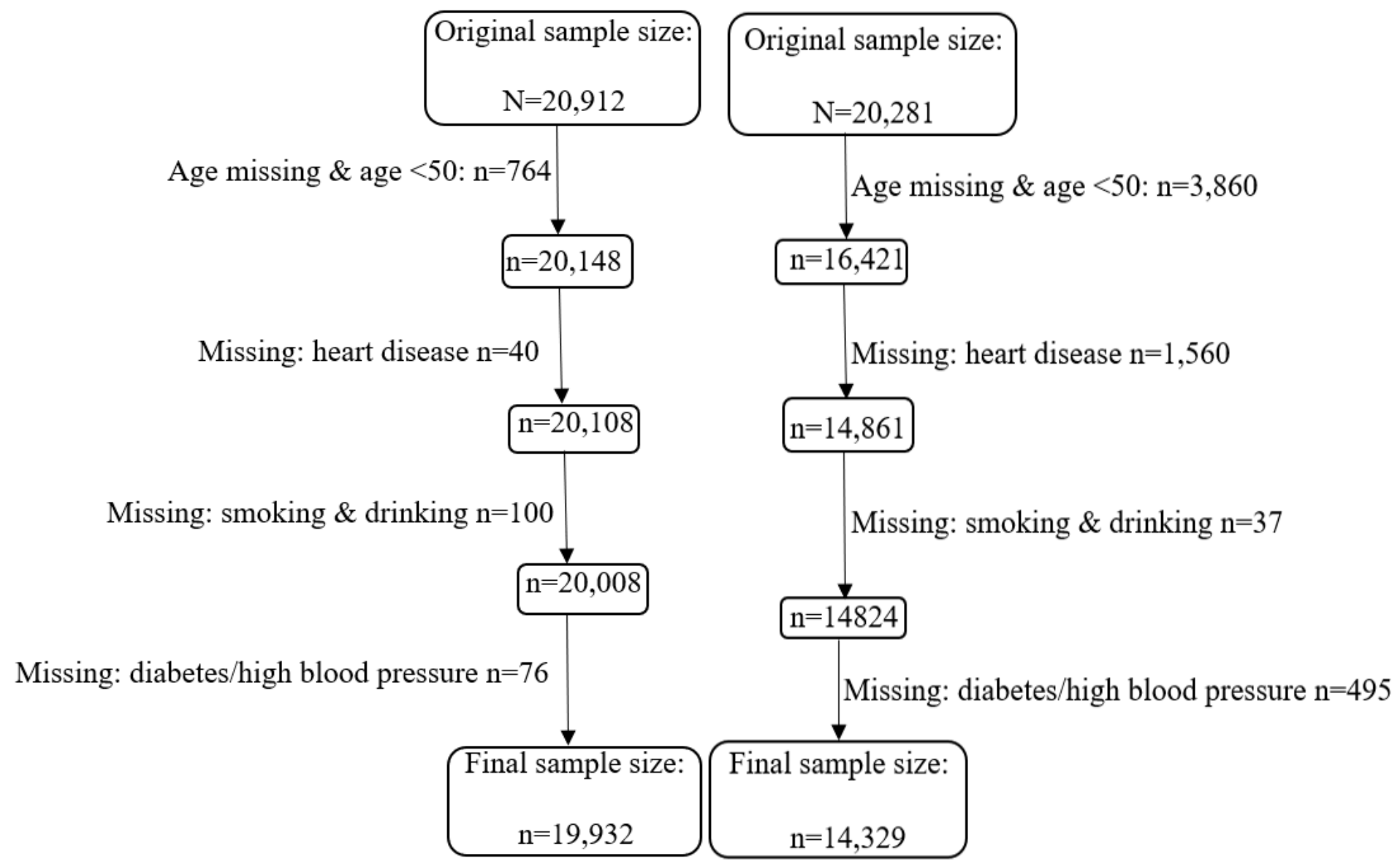

Figure 1

Analytical sample derived from 20,912 participants in the 2016 wave of HRS data (left) and 20,281 participants in the 2015 wave of CHARLS data (right). 


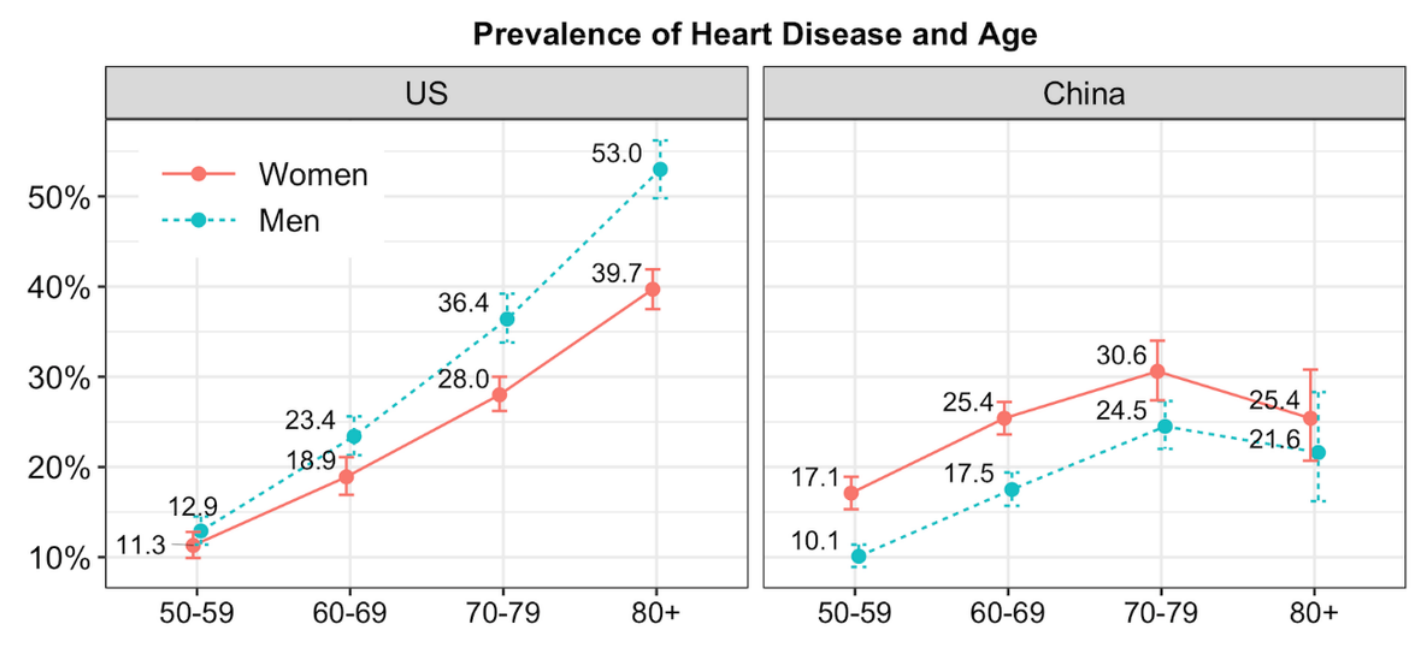

Prevalence of Heart Disease and Smoking/drinking behavior

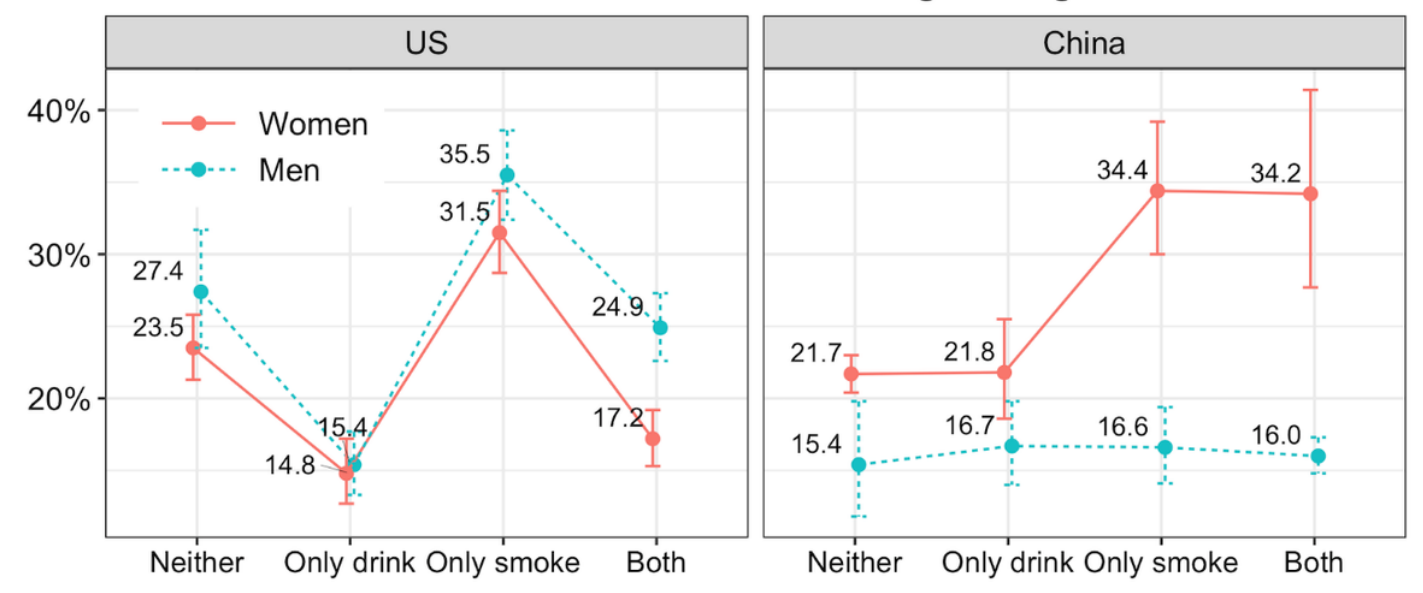

Figure 2

Weighted prevalences of heart disease by age and smoking/drinking behavior and corresponding $95 \%$ Cls. Non-overlapping $95 \%$ Cls between groups implies statistically significant differences.

\section{Supplementary Files}

This is a list of supplementary files associated with this preprint. Click to download.

- SupplementaryFiles.docx 